\title{
Thymic Hypoplasia in Japanese Black Calves with Stillbirth/Perinatal Weak Calf Syndrome
}

\author{
Masaki TAKASU ${ }^{1)}$, Kinji SHIROTA ${ }^{2)}$, Yasunori OHBA ${ }^{1 *}$, Naohito NISHII ${ }^{1)}$, Tetsuma MURASE ${ }^{1)}$, \\ Kiyoshi MIYAZAWA ${ }^{1)}$ and Hitoshi KITAGAWA ${ }^{1)}$ \\ ${ }^{1)}$ Department of Veterinary Medicine, Faculty of Applied Biological Sciences, Gifu University, 1-1 Yanagido, Gifu 501-1193 and \\ ${ }^{2)}$ Department of Veterinary Pathology, Faculty of Veterinary Medicine, Azabu University, 1-17-71 Fuchinobe, Sagamihara, Kanagawa \\ 229-8501, Japan
}

(Received 18 February 2008/Accepted 20 June 2008)

\begin{abstract}
To investigate the relationship between the decreased immunity associated with infirmity and low body weight at birth as a consequence of intrauterine growth retardation in Japanese Black calves with stillbirth/perinatal weak calf syndrome, the thymuses and spleens of 13 calves with this syndrome, weighing less than $20 \mathrm{~kg}$ at birth, were examined histopathologically. Cytokeratin staining of the thymus was also carried out to examine its composition. The thymus and spleen were classified as grades $0-4$ and I-III according to their hypoplasia, respectively. All calves showed a decreased number of thymocytes. One calf was classified as grade 1 , which was characterized by a starry sky appearance. Five calves were classified as grade 2, demonstrating a reversion of the cortex-to-medulla ratio, and the rest were classified as grades 3 and 4 showing an indistinguishable boundary between the cortex and medulla. The thymuses of grade 3 and 4 were occupied by stroma cells, and their Hassall bodies and other structures were rarely observed. Six of 13 calves showed a decreased number of splenocytes, grade II or III, and their red and white pulp regions were unclear. The intrauterine growth retardation caused by lack of growth factors during the fetal period might have induced thymic hypoplasia associated with decreased immunity in the calves with stillbirth/perinatal weak calf syndrome. Therefore, intrauterine growth retardation might be associated with one of the causes of decreased immunity involved in infirmity in this syndrome.

KEY WORDS: intrauterine growth retardation, Japanese Black cattle, low body weight, stillbirth/perinatal weak calf syndrome, thymic hypoplasia.
\end{abstract}

J. Vet. Med. Sci. 70(11): 1173-1177, 2008

In Japanese Black cattle, the mortality of neonates is estimated to be $4.5 \%$ [8], and approximately half of the neonates that died shortly after birth did not show any apparent causes of weakness. This so-called stillbirth/perinatal weak calf syndrome [10] is characterized by low body weight, anemia, depression, weakness, variable body temperature, a reddened and crusty muzzle, and astasia. These calves are highly susceptible to infection, and ultimately die showing diarrhea or symptoms of pneumonia within a few weeks after birth.

Japanese Black calves with stillbirth/perinatal weak calf syndrome have been suggested to have a congenital immune-insufficiency, especially an abnormality in systemic immunological function involving decreased CD8+ and $\gamma \mathrm{d} \mathrm{T}$ cells [9]. In fact, $\mathrm{T}$ cells play an important role in systemic immunity, and the $\mathrm{T}$ cell precursors derived from bone marrow undergo differentiation in the thymus. Therefore, the thymus is crucial to the immune system as a primary lymphoid organ $[2,6,11]$. Thus, the decreased immunity of calves with this syndrome might be associated with abnormality involving their thymus.

Calves with stillbirth/perinatal weak calf syndrome often have low body weights, less than $20 \mathrm{~kg}$ at birth [8], and the association of decreased immunity involved in infirmity and

\footnotetext{
* Correspondence to: Ohba, Y., Department of Veterinary Medicine, Faculty of Applied Biological Sciences, Gifu University, 11 Yanagido, Gifu 501-1193, Japan. e-mail: ohba@gifu-u.ac.jp
}

low weight at birth as a consequence of intrauterine growth retardation has been discussed [8]. However, there is no definite evidence of this. Therefore, we examined the pathological findings of primary lymphatic organs, the thymus and spleen, in Japanese Black calves that weighed less than $20 \mathrm{~kg}$ at birth.

\section{MATERIALS AND METHODS}

Calves: Thirteen Japanese Black calves with stillbirth/ perinatal weak calf syndrome weighing less than $20 \mathrm{~kg}$ at birth; 2 male, 8 female, and 3 sex-unknown calves, were examined in this study. The animals were neonates within 2 days (48 hr) after birth, including the stillborn. Since lymphatic regression, especially thymic regression, occurs when animals suffer from malnutrition, exhaustion, and infectious diseases $[2,5,7,11]$, the calves examined here were limited in order to eliminate postnatal influences upon them. Six calves were stillborn, and 7 calves died or were euthanized soon after birth. Six of them showed astasia, and only 2 calves suckled on their own. All calves were small in stature, and their average body weight at necropsy, which was carried out at $0.7 \pm 0.9$ days (mean \pm standard deviation) after birth, was $12.5 \pm 2.4 \mathrm{~kg}$. The gestation period of them was $285.8 \pm 6.5$ days, and this was considered normal. Their dams had been vaccinated against teratogenic infectious diseases; Aino, Akabane, Chuzan, and Ibaraki viruses, and they were free from bovine diarrhea (BVD) virus infec- 
tion which causes intrauterine growth retardation. In addition, the dams were kept under normal husbandry on commercial farms.

Tissue preparation and histological examination: Thymuses and spleens were collected, fixed with 10\% neutral buffered formalin, and embedded in paraffin. Specimens were cut at $4 \mu \mathrm{m}$ and stained with hematoxylin and eosin (HE). Additionally, immunohistochemistry [ABC procedure; Histofine SAB-PO (R) kit, Nichirei Biosciences, Tokyo.] of the thymus for cytokeratin staining (AE1/AE3 mouse monoclonal antibody, Dako, N3515, Kyoto. for primary antibody of cytokeratin) was carried out to examine the structure of the thymus.

Classifications of the thymus and spleen: The pathology of the thymus was classified grades $0-4$ according to the literature [13] as described below.

Grade 0: normal thymus. The density of the thymocytes in the cortex is higher than that in the medulla.

Grade 1: The thymocytes in the cortex exhibit pyknosis and/or karyorrhexis, and englobement is observed.

Grade 2: The number of thymocytes in the cortex decreases less than those in the medulla, and reversion of the cortex-to-medulla ratio is observed.

Grade 3: The boundary between the thymic cortex and medulla is indistinguishable.

Grade 4: Atrophy of the cortex is remarkable, or the thymocytes in the cortex disappear. This state is often observed under long-term stressful conditions, such as exhaustion or chronic infection.

The pathology of the spleen was classified based on the number of splenocytes as grades I (normal) to III (hypoplastic).

Tissues obtained from a male neonate weighing $34.5 \mathrm{~kg}$ were prepared as a histopathological control. This control calf died as a result of an accident and had no abnormalities.

\section{RESULTS}

Pathological findings of thymus: The gross findings of the thymus are shown in Fig. 1. Thymuses of the calves with stillbirth/perinatal weak calf syndrome were diminutive and thin, and some of them were indistinguishable from surrounding tissue. Moreover, they were divided into lobes, dispersed, and covered by a thin fibrous capsule of connective tissue.

The cortex and medulla of the thymus in the control calf was well-delineated, and the lobular structure was also clearly defined histologically (Fig. 2). However, most of the thymuses in calves with stillbirth/perinatal weak calf syndrome exhibited loss of total cell number, mainly thymocytes, and some of them demonstrated a barely identifiable boundary of the cortex and medulla. Immunohistochemistry of their thymuses clarified the decreased numbers of thymocytes. The thymuses of some calves were occupied by stroma cells, and their Hassall bodies and other structures of stroma cells were rarely observed.

Histological grade of thymic hypoplasia is shown in Fig. 3. One calf was classified as grade 1 , which was characterized by a starry sky appearance. Five of the 13 calves were classified as grade 2 thymic hypoplasia, a decreased number of thymocytes which induced a reversion of the cortex-tomedulla ratio. The rest demonstrated grades 3 and 4 thymic hypoplasia, showing an indistinguishable boundary between the cortex and medulla. Consequently, the pathology of the calves with stillbirth/perinatal weak calf syndrome was characterized by a decreased number of thymocytes.

Pathological findings of the spleen: The histological findings of the spleen are shown in Fig. 4. The total number of splenocytes was decreased in some calves with stillbirth/ perinatal weak calf syndrome, and their red pulp and white pulp regions were unclear. Fewer lymphocytes around the
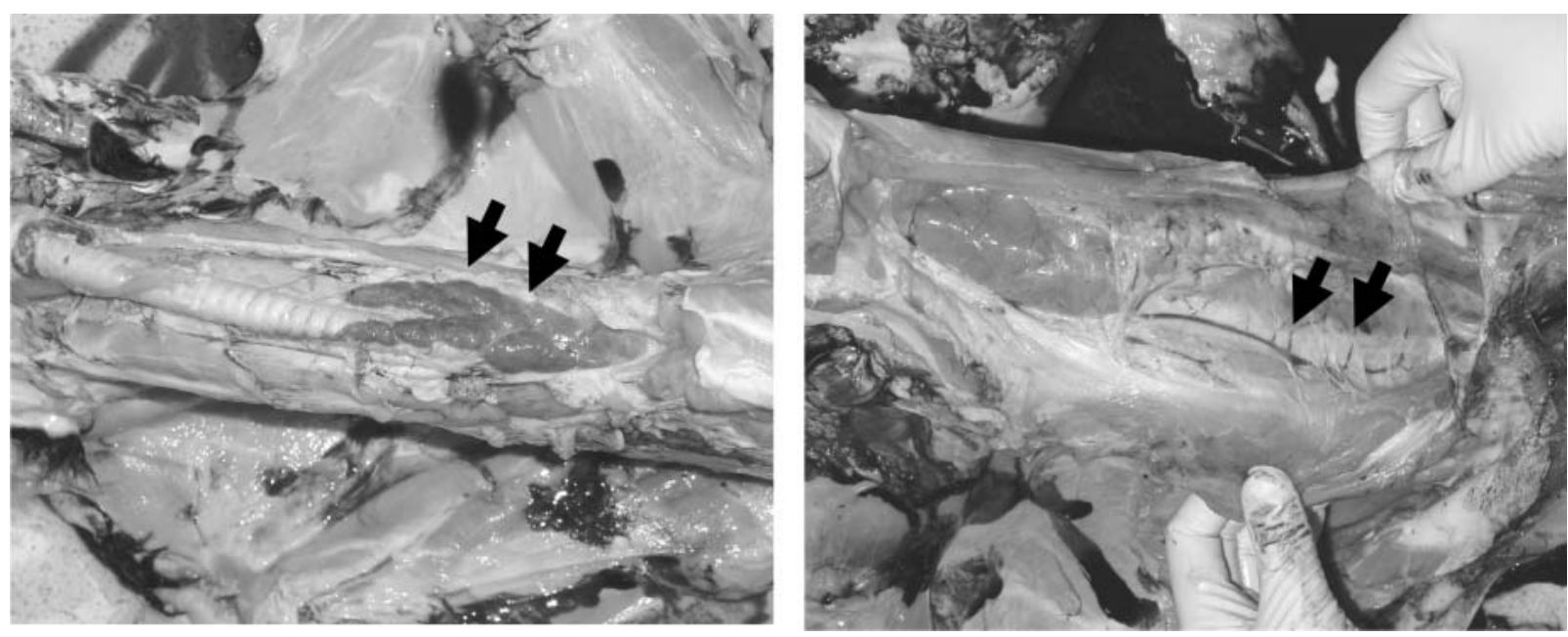

Fig. 1. Gross findings of the thymus in the control (left) and in a calf with stillbirth/perinatal weak calf syndrome (right). The neck part of the thymus in the control is well defined. However, the thymus of the calf with stillbirth/perinatal weak calf syndrome is diminutive and thin, and is indistinguishable from the surrounding tissue. Arrow: thymus. 

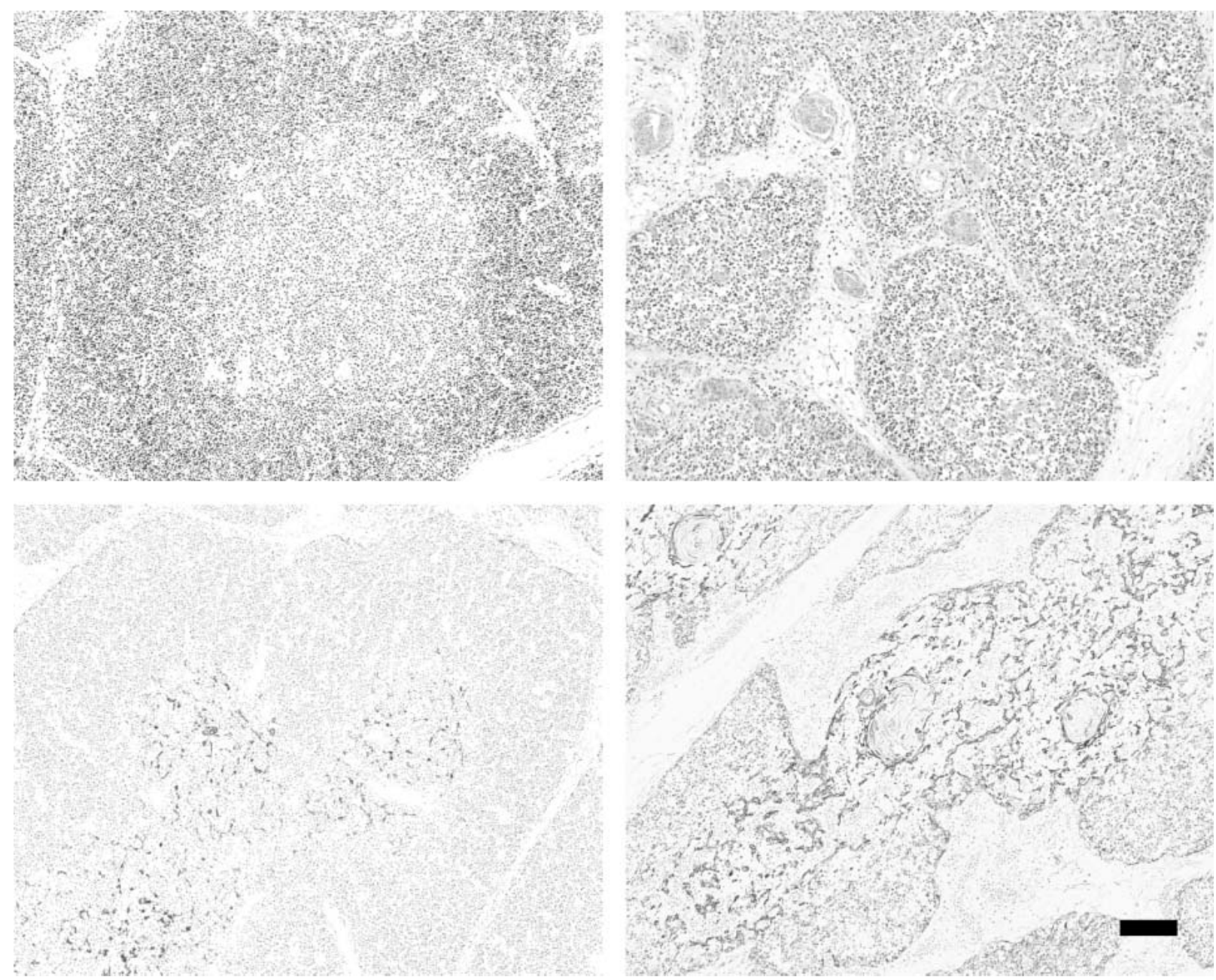

Fig. 2. Histological findings of the thymus in the control (left) and in a calf with stillbirth/perinatal weak calf syndrome (right). The cortex and medulla of the thymus in the control calf are well delineated, and the lobular structure is also clearly defined. However, the thymocytes in the cortex have disappeared, and the boundary of the cortex and medulla is hardly identifiable in the thymus of the calf with stillbirth/perinatal weak calf syndrome (grade 4). The cytokeratin staining identifies stroma cells from thymocytes. The thymus in the calf with stillbirth/perinatal weak calf syndrome is occuped by stroma cells, and the Hassall bodies and other structures of the stroma cells are rarely observed. Hematoxylin and eosin (upper) and cytokeratin staining (lower). Bar $=100 \mu \mathrm{m}$.

central artery in the white pulp and magnification of the sinus in the red pulp were observed in these calves, and stroma cells were clearly observed in both areas. Histological grade of splenic hypoplasia is shown in Fig. 3, and 6 of the 13 calves with stillbirth/perinatal weak calf syndrome had a decreased number of splenocytes, which was classified as grade II or III.

\section{DISCUSSION}

The calves with stillbirth/perinatal weak calf syndrome examined here demonstrated hypoplasia of lymphatic organs, especially thymus. They exhibited small numbers of thymocytes and abnormal structure of stroma cells, which might have caused difficulties in T cell differentiation, and this difficulties might have induce systemic immune-insuf- ficiency of the calves [4-7]. The thymus shows an extreme sensitivity to acute and chronic stressors inducing thymic involution, and glucocorticoid-induced thymocyte apoptosis is an important mechanism contributing to atrophy of the thymus under stress [2]. Moreover, the loss of thymocytes is an outcome of both acute and chronic protein malnutrition, which seems to result from enhanced thymocyte death and decreased thymocyte proliferation [11]. However, these factors which induce thymic hypoplasia, excessive stress and neonatal malnutrition after birth, were eliminated by careful selection of the subjects in this study. Thus, the congenital factors of thymic hypoplasia observed here should be existed.

The congenital factors that influence thymic hypoplasia are intrauterine infection during the fetal period and intrauterine growth retardation [1]. However, all the pregnant cat- 


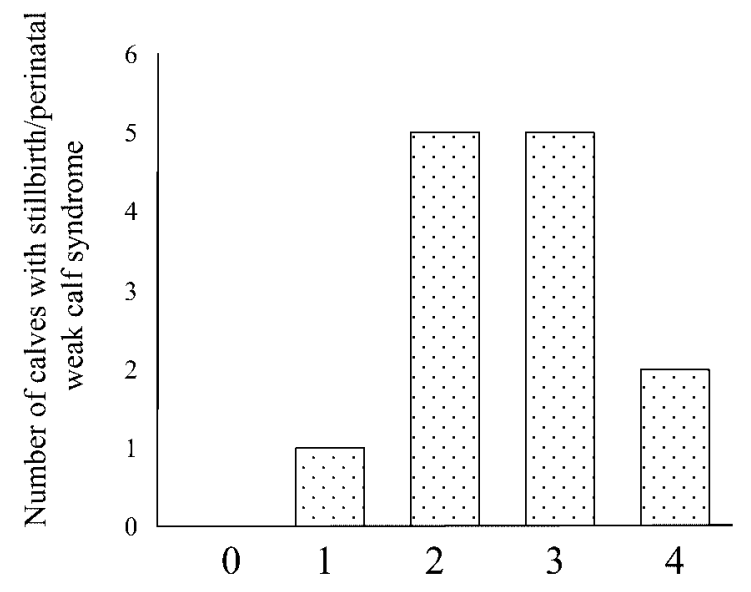

Histological grade of thymic hypoplasia

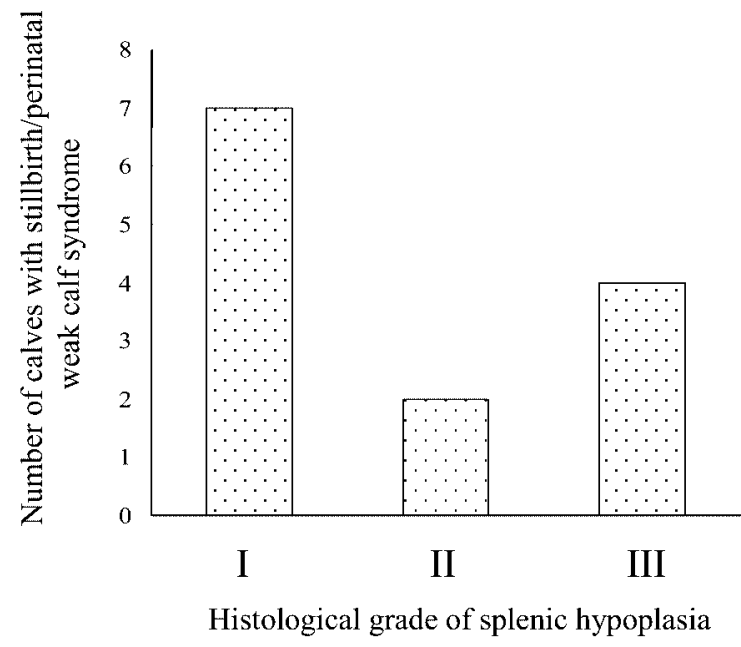

Fig. 3. Histological classification of the thymuses (left) and spleens (right) in calves with stillbirth/perinatal weak calf syndrome. The pathophysiology of the thymus was classified as grade 0 to grade 4 according to the literature, and that of the spleen was graded as normal (grade I) to hypoplastic (grade III).
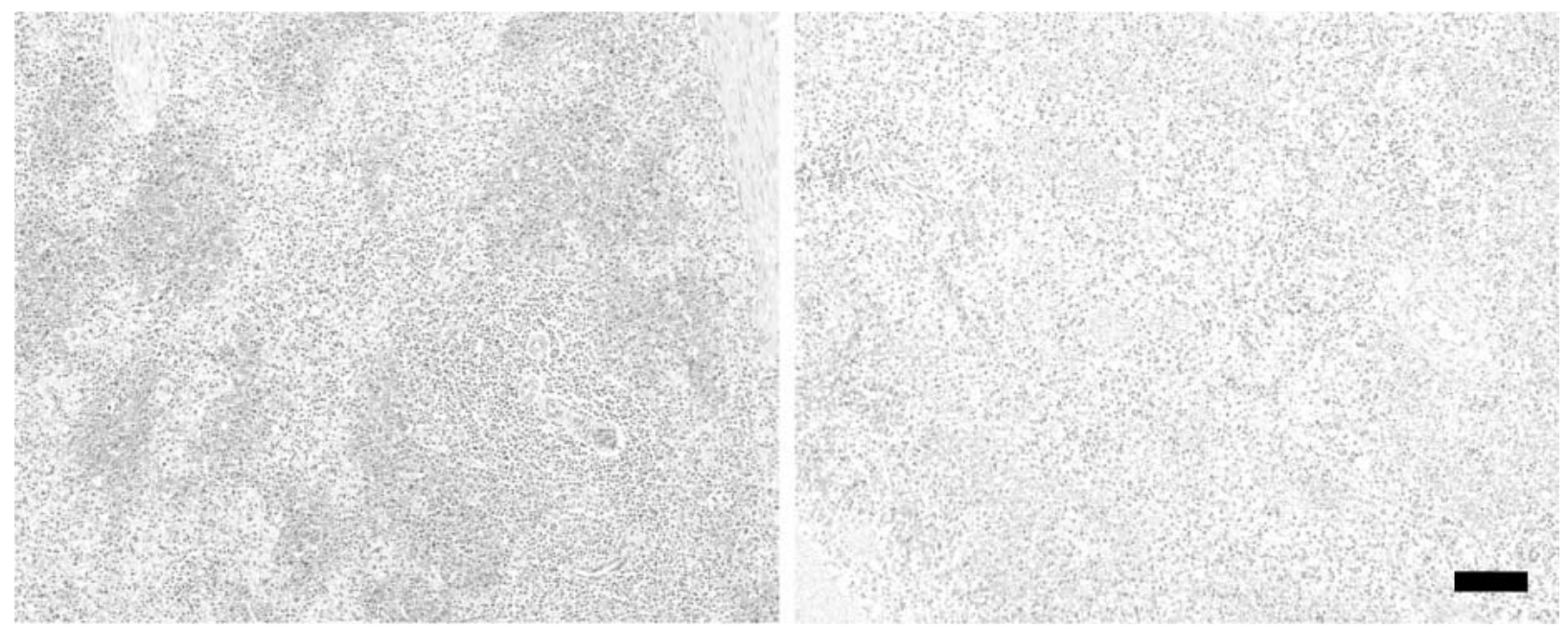

Fig. 4. Histological findings of the spleen in the control (left) and in a calf with stillbirth/perinatal weak calf syndrome (right). Both the red and white pulp regions of the spleen in the control calf are well delineated. However, the spleen in the calf with stillbirth/perinatal weak calf syndrome is classified as grade III, which is loss of total number of splenocytes, and the red and white pulp regions are unclear. Hematoxylin and eosin staining. Bar=100 $\mu \mathrm{m}$.

tle in our area had been vaccinated against teratogenic viral infections, and the dams of the calves with stillbirth/perinatal weak calf syndrome in this study were free of BVD virus infection. Therefore, there was less possibility that teratogenic viral infection caused the thymic hypoplasia in the calves with stillbirth/perinatal weak calf syndrome, and thus the other cause of thymic hypoplasia, that is intrauterine growth retardation, should be existed.

The cause of intrauterine growth retardation is said to be maternal malnutrition, placental insufficiency, and low expression of fetal growth factors $[3,11,12]$. Unfortunately, it was impossible to determine the cause of intrauterine growth retardation in this study. However, all these causes of intrauterine growth retardation induce low expression of the fetal growth factors involved in normal fetal growth $[3,11,12]$, and these growth factors are also involved in development of lymphatic organs including thymus during the fetal period. In fact, growth restriction of fetal sheep by placentectomy in their dam decreases the weights and sizes of their thymuses and other lymphatic organs [1]. Moreover, pulsatile infusion of a growth hormone that increases fetal growth factors into the fetus with growth restriction increases the weight and size of the thymus and other lymphatic organs, while the untreated growth restricted fetuses were smaller than those of normal sheep [1]. In addition, growth factors such as insulin-like growth 
factor-1 may serve to increase migration and colonization of bone marrow-derived precursor cells to the thymus and other lymphatic organs. Therefore, the lack of growth factors during the fetal period, in other words, intrauterine growth retardation, might have induced decreased immunity, especially thymic hypoplasia, associated with infirmity in calves with stillbirth/perinatal weak calf syndrome.

Consequently, the intrauterine growth retardation caused by lack of some growth factors during fetal period might have induced thymic hypoplasia associated with decreased immunity in calves with stillbirth/perinatal weak calf syndrome. Therefore, although there are numerous causes of stillbirth/perinatal weak calf syndrome, intrauterine growth retardation might be associated with one of the causes of decreased immunity associated with infirmity in this syndrome.

\section{REFERENCES}

1. Bauer, M. K., Breier, B. B., Bloomfield, F. H., Jensen, E. C., Gluckman, P. D. and Harding, J. E., 2003. Chronic pulsatile infusion of growth hormone to growth-restricted fetal sheep increases circulating fetal insulin-like growth factor-I levels but not fetal growth. J. Endocrinol. 177: 83-92.

2. Engler, H. and Stefanski, V. 2003. Social stress and T cell maturation in male rats: transient and persistent alterations in thymic function. Psychoneuroendocrinology 28: 951-969.

3. Fisher, J. W. 2003. Erythropoietin: physiology and pharmacology update. Exp. Biol. Med. 228: 1-14.

4. French, R. A., Broussard, S. R., Meier, W. A., Minshall, C., Arkins, S., Zachary, J., Dantzer, F. R. and Kelley, K. W. 2002. Age-associated loss of bone marrow hematopoietic cells is reversed by GH and accompanies thymic reconstitution. Endocrinology 143: 690-699.
5. Malpuech-Brugere, C., Nowacki, W., Gueux, E., Kuryszko, J., Rock, E., Rayssiguier, Y. and Mazur, A. 1999. Accelerated thymus involution in magnesium-deficient rats is related to enhanced apoptosis and sensitivity to oxidative stress. Br. J. Nutr. 81: 405-411.

6. Montecino-Rodriguez, E., Clark, R. and Dorshkind, K. 1998. Effects of insulin-like growth factor administration and bone marrow transplantation on thymopoiesis in aged mice. Endocrinology 139: 4120-4126.

7. Nodera, M., Yanagisawa, H. and Wada, O. 2001. Increased apoptosis in a variety of tissues of zinc-deficient rats. Life Sci. 69: 1639-1649.

8. Ogata, Y., Nakao, T., Takahashi, K., Abe, H., Misawa, T., Urushiyama, Y. and Sakai, J. 1999.Intrauterine growth retardation as a cause of perinatal mortality in Japanese Black beef calves. Zentralbl. Veterinärmed. A 46: 327-334.

9. Ohtsuka, H., Fukunaga, N., Hara, H., Fukuda, S., Hayashi, T., Hoshi, F., Yoshino, T., Koiwa, M. and Kawamura, S. 2003. Changes in peripheral leukocyte populations of weak calf syndrome of Japanese Black calves. J. Vet. Med. Sci. 65: 793-796.

10. Radostits, O. M., Gay, C. C., Blood, D. C. and Hinchcliff, K. W. 2000. Specific diseases of uncertain etiology. pp. 17631766. In: Veterinary Medicine, W.B. Saunders, London.

11. Savino, W. 2002. The thymus gland is a target in malnutrition. Eur. J. Clin. Nutr. 56 (Suppl. 3): S46-49.

12. Smaniotto, S., Ribeiro-Carvalho, M. M., Dardenne, M., Savino, W. and de Mello-Coelho, V. 2004. Growth hormone stimulates the selective trafficking of thymic CD4+CD8- emigrants to peripheral lymphoid organs. Neuroimmunomodulation 11: 299-306.

13. Tamaki, K. 1987. Development of thymus: structure, function, and its abnormality. pp. 398-405. In: Compendium of Current Pathology 18B (Iijima, S. ed.), Nakayama Shoten. (in Japanese). 